\title{
Intelligent Wireless Surveillance of Multirate Induction Motors
}

\author{
M. Nouby \\ South Valley University, \\ Qena, Egypt \\ Nouby.ghazaly@eng.svu.edu.eg
}

\begin{abstract}
Recent advances in the automation field provide a wide range of scope in control and monitoring of the industrial systems. A very accurate automation system is on high demand in recent times. This paper mainly deals about remote monitoring and control of the induction motor. The main objective is to develop an embedded system to prevent the occurrence of line fault in a three phase system (say induction motor) and to display and control the parameters voltage, speed and temperature of the three phase system using Zig-bee communication technology. An ARM microcontroller is used in this project, which controls the entire operation of the system, which also communicates various parameters from one part of the system to other through Zig-bee technology. To prevent the issues of poor blunder elements and commotion affectability emerging in functional applications, the plan issue is changed into a proportional Linear Matrix Inequality (LMI) structure to understand a vigorous state criticism roughly by quick yield testing. The control objective is encircled with rise time, overshoot and settling time particulars. Subsequent to arriving at the consistent express the controller ought to look after solidness. To fulfill the above prerequisites a quick yield inspecting control calculation is planned with the end goal that the ideal shut circle conduct and shut circle soundness is accomplished. The exhibition of the framework is broke down for controlling the speed through wireless networks. .
\end{abstract}

Keywords-Zig-bee, ARM, LMI, wireless communication

\section{Introduction}

As the quick improvement of 21 st century social economy, the remote correspondence request is expanding angrily in the mean time the concerned method is continuing emerging. The remote correspondence advancements are quickly spreading to numerous new zones, including the mechanization and the significance of the utilization of remote innovations in the information obtaining, building control, observing frameworks and robotization of assembling forms are of high intrigue. The utilization of remote advancements for short separation mechanical interchanges has as of late pulled in developing interest as a result of the perceived favorable circumstances of these innovations like portability, lower cost, arrangement adaptability, data transmission fit to the qualities of correspondences between modern components, utilization of recurrence groups not requiring managerial approval, and so forth. Every one of these focal points are clearly gotten without weakening data move security. The zig-bee convention has as of late come to be viewed as a decent possibility for use in modern situations [1][2].

Because of security and affordable reasons, checking and symptomatic frameworks are of developing enthusiasm for every single modern application. An expanding number of utilizations require having the option to identify potential flaws emerging during the typical movement of the electronic framework, thus, on-line shortcoming identification is a hotly debated issue today. Estimating the different parameters of a mains power gracefully arrange has become a vital requirement for both modern procedures and in house hold forms [3].

Increasingly pivoting machines are key parts and touchy in a few force plants. Industry requires electric engines to be ensured against harms. Fixing or rewinding an electric engine can be an exorbitant occasion however personal time could be considerably increasingly expensive. Early alerts can wipe out the loss of creation time and exorbitant engine fix. Warm assurance is one of the most significant angles in engine condition observing. $30 \%$ of engine disappointments are identified with stator winding protection. It is ordinarily expected that the engine's life is decreased by half for each $10{ }^{\circ} \mathrm{C}$ increment over its stator winding temperature limit Along these lines, exact checking of the stator winding temperature is pivotal for engine insurance purposes [4].

These days, squirrel-confine acceptance engines are broadly utilized in electromechanical actuators since they have a notoriety of strength and harshness. Besides, the ease supports its utilization in contrast with different sorts of engine. On the off chance that the enlistment engine is a significant component in the mechanical procedure, we have to make early blame recognition. This one comprises in constraining the expense, or restricting the hazardousness of the deficient procedure. Hence, a checking framework must be made realizing that this one keeps on working ordinarily 
[5]. By considering all the critical factors mentioned the embedded system to be developed is capable of detecting the occurrence of line faults, protecting the induction motor against thermal problems, controls and monitors the speed with the help of zig-bee wireless communication technology [6].

\section{EXISTING TECHNIQUES}

DC motor drives are still commonly used in various ventures, for instance, moving plants, paper machines, and slackening up and rewinding machines [7]. DC motor drives go about as an interface between a controller and the motor. The drive must match the control signals (voltage and power levels) and sign sort (straightforward or progressed). The drive produces power change, upgrade, and sequencing of waveform signals. Sorts of DC motor drives fuse adaptable or variable speed control, servo control, and essential development control. Some early DC motor drives used fixed-speed AC motors to drive $\mathrm{DC}$ generators that made the variable voltage for the DC motor. Nowadays, the DC motor drives generally speaking work from a solid state power deftly, and since this power effortlessly is usually more monetarily smart than the motor generator set. The DC motor drive can be more direct and more affordable than the variable repeat drive, regardless, when the more prominent cost of the DC motor is fused [8].

The states of the structure are required for the info. Here the estimation of the information is problematic. So the cost augmentations and diminishes the unflinching nature of the structure. Slight assortments of the parameter from the apparent worth may result into basic debasement of the shut circle execution. In case the state of the structure isn't open for direct estimations it is routinely possible to choose the state by considering the state through our knowledge into the components and progressively compelled estimations. This is done by building a spectator that uses the estimations of the data and yields of an immediate structure, nearby a model of the system components, to assess the state [9].

Yield analysis controller is realized by discrediting a controller organized under interminable time state input and using a discrete-time high-gain onlooker to assess the system states. It was demonstrated that the yield input controller settles the beginning stage of the shut hover system for sufficiently small testing period and recovers the show under relentless time state analysis as the investigating repeat is extended. For the discrete-time high-gain onlooker dynamically precise estimation of the structure states is cultivated by snappier examining of the yield. We introduced a multirate yield analysis control plot for a class of nonlinear systems subject to discrete-time high expansion observers.
Onlooker based controller setup crushes the issues of ordinary spatial control systems organized subject to state analysis rule. The major issue of the passerby based analysis controller is the supposed division issue. It infers that a controller and a passerby can be arranged freely, so the joined observer controller yield analysis spare the essential features of the controller with the full is state open. The separation rule was shown for asymptotic perpetual info modification of a class of nonlinear systems got together with high-gain onlookers.

In any case, the passerby based arrangement fabricates the use cost and diminishes steadfastness of control structure. In this way it is appealing to go for a yield input structure. If the yield is open, by then the yield info can be used to structure the controller. Yield input controllers are of static and dynamic. The static yield analysis controller is one of the most investigated issues in control speculation and application. In any case a critical imperative of this strategy is that the security of the shut structure isn't guaranteed. It fuses computational ease, action solely with quantifiable sums, execute limit in constrained time, doesn't exist for all controllable and noticeable, no additional states to the state of development in straight system. It is less sensitive to execute and progressively reliable. The dynamic yield input controller incorporates progressively amazing and is staggering to design. Multirate yield analysis is trying the control information and sensor yield of the structure at different rates [10].

Rather than onlooker based arrangement in which accuracy of estimation of states improves after long interval of time, cautious computation of states in just one looking at period is conceivable if multirate yield input is used. In this system, the yield gains are piecewise steady and the controller can be easily executed. It furthermore guarantees the adequacy of the shut circle system and doesn't use present information or respect figure the states, consequently obliging time concede required for control law use. In this Multirate yield analysis controller there are two sorts. Speedy Output Sampling (FOS) and Periodic Output Sampling (POS). In Periodic Output Feedback control count, a sort of multirate yield input (MROF), the system yield is assessed at a moderate rate and control is invigorated at a speedy rate. This control count needn't waste time with any assumptions foresee that the need of the structure to be absolutely controllable and conspicuous. No state estimation is required since the yield is in the analysis way, not the states. Further, this control computation has extraordinary closed circle dauntlessness and addresses least complex shut circle control which can be recognized in practical structures. This control figuring can give the perfect execution even inside seeing unmeasured disrupting impacts. 


\section{Proposed Methodology}

The systems input is a three phase $\mathrm{R}, \mathrm{Y}, \mathrm{B}$ voltage, which is going to be the input to the induction motor. A potential transformer is connected to the three phase supply, which senses the supply voltage and the corresponding signals are given to the ARM processor. The ARM processor will have LCD display unit connected to it, which is capable of displaying the sensed voltage from the three phase supply lines $\mathrm{R}, \mathrm{Y}, \mathrm{B}$. A single phase preventer is connected to the ARM processor.

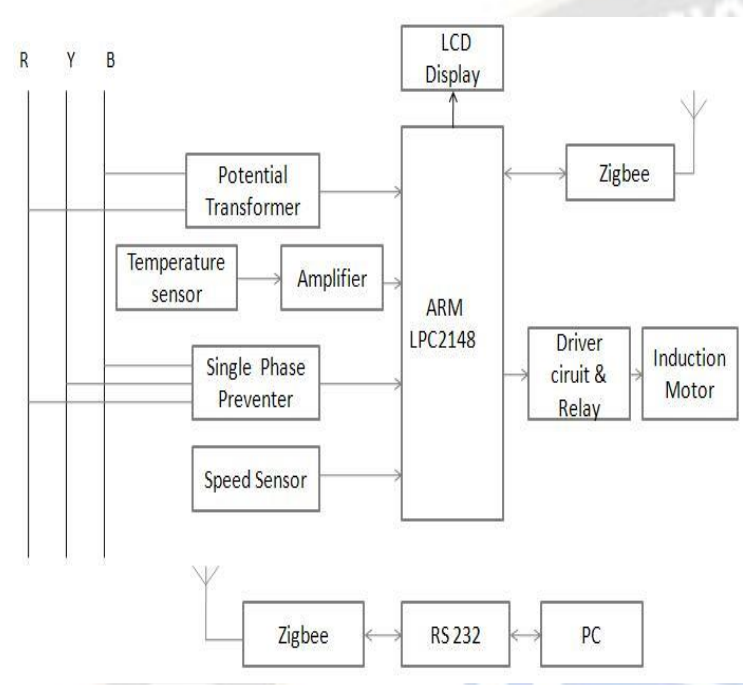

Fig 1: Structure of the proposed system

The occurrence of fault in three phase system may occur at any time due to many reasons. The main purpose of the single phase preventer circuit is to prevent single phasing or any other supply line fault. The single phase preventer's output will be given to the ARM processor, which will drive the induction motor accordingly. By this way the fault occurrence in the supply lines can be prevented before damaging the coil of the induction motor.

Zig-bee is another remote innovation created by the Zig-bee Alliance to beat the constraints of BLUETOOTH and Wi-Fi. The Zig-bee Alliance is a relationship of organizations cooperating to empower dependable, practical, low-power, remotely arranged, observing and control items dependent on an open worldwide norm. The objective of the Zig-bee Alliance is to furnish the shopper with extreme adaptability, versatility, and convenience by building remote insight and capacities into consistently gadgets. Zig-bee is created on the highest point of IEEE 802.15.4 norm. It is intended for lowpower utilization permitting batteries to basically keep going forever.

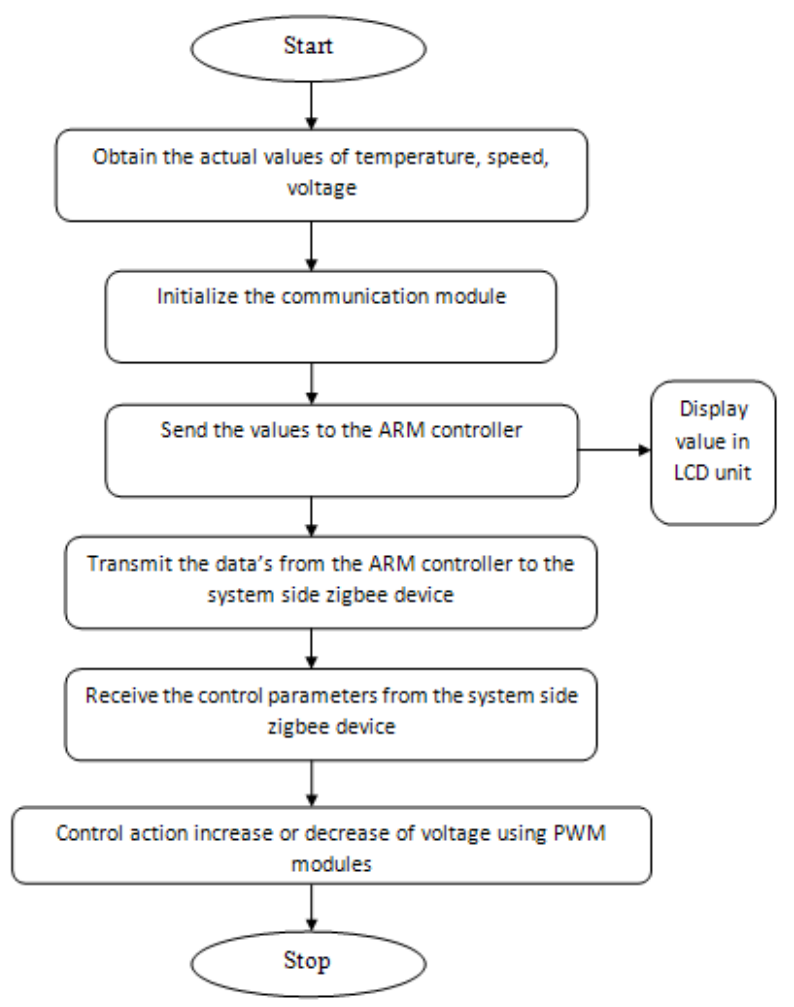

Fig 2: Flowchart of proposed system

The name Zig-bee is said to originate from the residential bumble bee which utilizes a crisscross kind of move to impart significant data to different individuals. This correspondence move (the "Zig-bee Principle") is the thing that architects are attempting to copy with this convention, a lot of independent and basic living beings that combine to handle complex errands. Zig-bee gadgets permit batteries to last up to years utilizing essential cells (minimal effort) with no chargers. The forthcoming of Zig-bee will upset the home systems administration and rest of the remote world. Along these lines, Zig-bee innovation is a low information rate, low force utilization, minimal effort; remote systems administration convention focused towards robotization and remote control applications.

\section{Simulation Results}

A speed sensor circuit will sense the speed of the induction motor. The speed values are given to the ARM processor, which will be displayed in the LCD display unit. The speed values are stored in the ARM processor and those values are compared with the user defined values of speed, which are set through the PC. Zig-bee communication enables wireless duplex transmission of temperature, voltage and speed values between the ARM processor and the PC. A RS232 serial communication is established between the PC and zig-bee module. According to the values set by the user for speed, voltage and temperature, corresponding control action will decided by the ARM processor. The ARM processor drives 
the induction motor through a driver and relay circuit. The simulations through simulink are shown in figure 3.

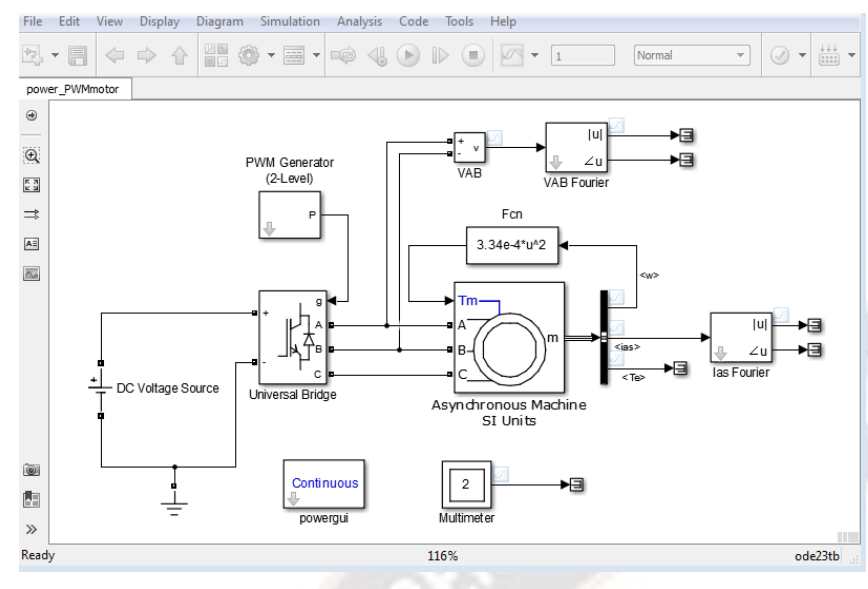

Fig 3: Simulation result for monitoring of induction motor

In the case of armature current analysis, the open loop response is taken for step input which gave the rise time as $0.28 \mathrm{sec}$. and the close loop response is obtained by applying state feedback and output feedback. In the state feedback, rise time is $0.02 \mathrm{sec}$, settling time is $-0.025 \mathrm{sec}$ and the gain is [6.7978 0.1539] as similar the output feedback has obtained the gain as [-3.9757 3.9787] here the steady state error is obtained as $0.5 \mathrm{sec}$.In the case of Angular velocity analysis, the open loop response is taken for step input which gave the rise time as $0.113 \mathrm{sec}$, and the close loop response is obtained by applying state feedback and output feedback.

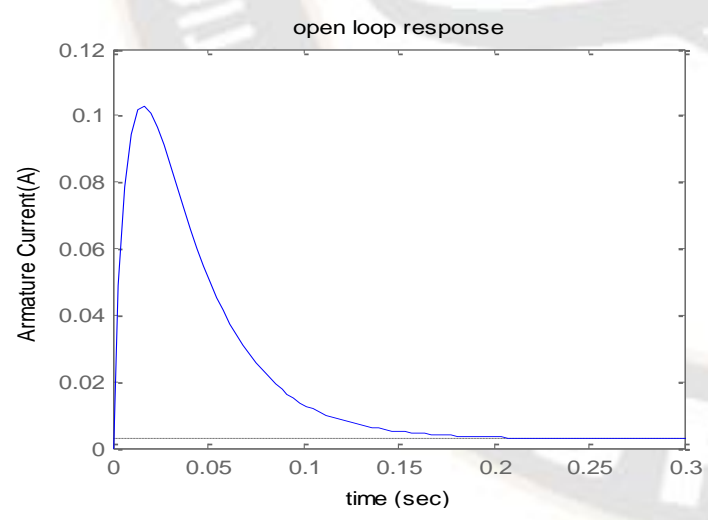

Fig 4: Open loop response for Armature current

In the state feedback, rise time is $0.18 \mathrm{sec}$, settling time is $0.166 \mathrm{sec}$ and the gain is [6.7978 0.1539] as similar the output feedback has obtained the gain as[-98.1270 103.4472] here the steady state error is obtained as $0.5 \mathrm{sec}$. This is shown in figure 5.

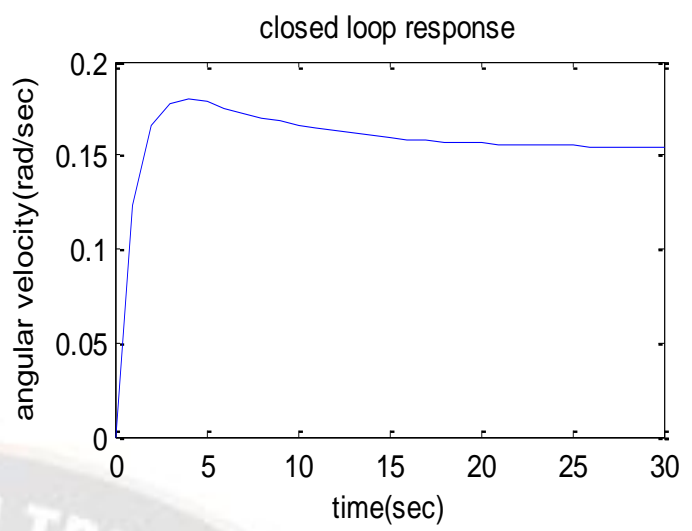

Fig 5: Closed loop response for angular velocity

The enlistment engine is associated with the microcontroller through the driver circuit. There is a transmitter and recipient side for the X-Bee module. Despite the fact that there is order of transmitter and recipient, the two modules will perform both transmitting and getting activity. The one section is associated with the controller, while the other part is associated with the framework part, where the set estimations of the parameter are resolved and checking of the parameter esteems are done. Now the transistor is directing, because of that authority and producer terminal is shorted. The 40106 schmitt trigger inverter is associated in the gatherer terminal. So zero voltage is given to inverter information and $+5 \mathrm{v}$ is taken in the inverter yield which is given as contribution to 7410 AND door. On the off chance that three line voltage is available, all contributions to the AND entryway is high which is given to microcontroller or other interfacing circuits.

\section{Table I COMPARISON OF THE CLOSED LOOP AND OPEN LOOP OF THE PROPOSED SYSTEM}

\begin{tabular}{|l|l|l|l|}
\hline $\begin{array}{l}\text { Angular } \\
\text { velocity }\end{array}$ & $\begin{array}{l}\text { Rise } \\
\text { time(sec) }\end{array}$ & $\begin{array}{l}\text { Settling } \\
\text { time (sec) }\end{array}$ & Gain \\
\hline Open loop & 0.113 & 0.145 & 4.214 \\
\hline $\begin{array}{l}\text { Closed } \\
\text { loop(SFB) }\end{array}$ & 0.183 & 0.166 & 6.7978 \\
\hline $\begin{array}{l}\text { Closed } \\
\text { loop(OFB) }\end{array}$ & 0.241 & 0.241 & 103447 \\
\hline
\end{tabular}

\section{Conclusion}

The proposed methodology includes hardware components for controlling the entire operation, a single phase preventer , a temperature sensor and a speed sensor module for measuring the temperature and speed respectively. The temperature and speed values are analog values and are given to the A/D pin of the ARM controller. The zigbee device used is $\mathrm{X}$-Bee. The XBee ZNet 2.5 modules used are more advanced than the popular XBee Series 1 modules. The model developed will prevent the occurrence of single phase 
in the induction motor, controls and monitors the temperature speed and voltage parameters. The zigbee communication module used in this paper enables efficient means of wireless communication. Thus the prototype model developed can be used in any industrial processes where short range wireless communication can be implemented. Simulation result shows better efficiency than existing methods.

\section{References}

[1] Shizhuang Lin, Jingyu Liu, Yanjun Fang.“ ZigBee Based Wireless Sensor Networks and Its Applications in Industrial[C],'IEEE International Conference on Automation and Logistics, 2007 .pp:1979 - 1983.

[2] Jin-shyan Lee and Yang-chih Huang, Design and implementation of ZigBee/IEEE 802.15.4 Nodes for Wireless Sensor Networks, 2006.

[3] Daniel E. Viassolo, Mario A. Rotea "Practical Design Of Multirate Output Controllers" Proceedings of the 37 th IEEE conference on decision and control December 1988

[4] Xin Ying, Xie Guangzhong, Jiang Yadong, Wireless temperature sensor network based on ZigBee protocol, sensor and microsystem, 2006, 7(25): pp.82-84.

[5] Sunil oberoi and Bandyopadhyay "Robust control of a laboratory scale launch vehicle model using fast output sampling technique" Proceedings of 5 Th Asian control conference 2004

[6] Egan, "The Emergence of ZigBee in Building Automation and Industrial Controls". IEE Computing \& Control Engineering, Vol. 16, No. 2, pp. 14-19, April/May 2005.

[7] Sun Jinsheng, Wang Ning and Liu Liping, "Using ZigBee Wireless Network to Transfer Water-Sludge Interface Data" Information Acquisition, 2006 IEEE International Conference on Aug. 2006 Page(s):473 - 477.

[8] "ZigBee Based Wireless Sensor Networks and Its Applications in Industrial" Automation and Logistics, 2007 IEEE International Conference on 18-21 Aug. 2007 Page(s): $1979-1983$.

[9] A. Bhattacharjee, G. Ghosh, V. Kumar Tayal and P. Choudekar, "Speed control of BLDC motor through mobile application via secured Bluetooth," 2017 Recent Developments in Control, Automation \& Power Engineering (RDCAPE), Noida, 2017, pp. 203-206, doi: 10.1109/RDCAPE.2017.8358267.

[10] C. Vasilescu, V. Popescu, G. Varzaru, B. Mihailescu and I. Plotog, "Remote control of sensor-less permanent magnet synchronous motor," 2017 9th International Conference on Electronics, Computers and Artificial Intelligence (ECAI), Targoviste, 2017, pp. 1-4, doi: 10.1109/ECAI.2017.8166467. 